\title{
Patient-Centered, Payer-Centered, or Both? The 30-Day Readmission Metric
}

\author{
Craig A. Umscheid, MD, MSCE ${ }^{1,2,3,4 *}$, S. Ryan Greysen, MD, MHS, MA 3,4
}

${ }^{1}$ Center for Evidence-based Practice, University of Pennsylvania Health System, Philadelphia, Pennsylvania; ${ }^{2}$ Department of Biostatistics, Epidemiology and Informatics, University of Pennsylvania Perelman School of Medicine, Philadelphia, Pennsylvania; ${ }^{3}$ Department of Medicine, University of Pennsylvania Perelman School of Medicine, Philadelphia, Pennsylvania; ${ }^{4}$ Leonard Davis Institute of Health Economics, University of Pennsylvania, Philadelphia, Pennsylvania.

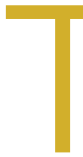

here is little doubt that preventing 30-day readmissions to the hospital results in lower costs for payers. However, reducing costs alone does not make this metric a measure of "high value" care. ${ }^{1}$ Rather, it is the improvement in the effectiveness of the discharge process that occurs alongside lower costs that makes readmission reduction efforts "high value" - or a "win-win" for patients and payers.

However, the article by Nuckols and colleagues in this month's issue of the Journal of Hospital Medicine (JHM) suggests that it might not be that simple and adds nuance to the ongoing discussion about the 30 -day readmission metric. ${ }^{2}$ The study used data collected by the federal government to examine changes not only in 30-day readmission rates between 2009-2010 and 2013-2014 but also changes in emergency department (ED) and observation unit visits. What they found is important. In general, despite reductions in 30-day readmissions for patients served by Medicare and private insurance, there were increases in observation unit and ED visits across all payer types (including Medicare and private insurance). These increases in observation unit and ED visits resulted in statistically higher overall "revisit" rates for the uninsured and those insured by Medicaid and offset any improvements in the "revisit" rates resulting from reductions in 30-day readmissions for those with private insurance. Those insured by Medicarerepresenting about 300,000 of the 420,000 visits analyzed-still had a statistically lower "revisit" rate, but it was only marginally lower (25.0\% in 2013-2014 versus 25.3\% in 2009-2010). ${ }^{2}$

The generalizability of the Nuckols' study was limited in that it examined only index admissions for acute myocardial infarction (AMI), heart failure (HF), and pneumonia and used data from only Georgia, Nebraska, South Carolina, and Tennessee-the four states where observation and ED visit data were available in the federal database. ${ }^{2}$ The study also did not examine hospital-level revisit data; hence, it was not able to determine if hospitals with greater reductions in readmission rates had greater increases in observation or ED visits, as one might predict. Despite these limitations, the rigor of the study

*Address for correspondence: Craig A. Umscheid, MD, MSCE, Perelman Center for Advanced Medicine, South Pavilion, 6th Floor, Office 623, 3400 Civic Center Boulevard, Philadelphia, PA 19104; Telephone: (215) 349-8098; Fax: (215) 349-8232; E-mail: craig.umscheid@uphs.upenn.edu

Received: March 5, 2018; Accepted: March 11, 2018

2018 Society of Hospital Medicine DOI 10.12788/jhm.2989 was noteworthy. The authors used matching techniques to ensure that the populations examined in the two time periods were comparable. Unlike previous research, ${ }^{3,4}$ they also used a comprehensive definition of a hospital "revisit" (including both observation and ED visits) and measured "revisit" rates across several payer types, rather than focusing exclusively on those covered by fee for service Medicare, as in past studies. ${ }^{4,5}$

What the study by Nuckols and colleagues suggests is that even though patients may be readmitted less, they may be coming back to the ED or getting admitted to the observation unit more, resulting in overall "revisit" rates that are marginally lower for Medicare patients, but often the same or even higher for other payer groups, particularly disadvantaged payer groups who are uninsured or insured by Medicaid. ${ }^{2}$ Although the authors do not assert causality for these trends, it is worth noting that the much-discussed Hospital Readmission Reduction Program (or "readmission penalty") applies only to Medicare patients aged more than 65 years. It is likely that this program influenced the differences identified between payer groups in this article.

Beyond the policy implications of these findings, the experience of patients cared for in these different settings is of paramount importance. Unfortunately, there are limited data comparing patient perceptions, preferences, or outcomes resulting from readmission to an inpatient service versus an observation unit or ED visit within 30 days of discharge. However, there is reason to believe that costs could be higher for some patients treated in the ED or an observation unit as compared to those in the inpatient setting, ${ }^{6}$ and that care continuity and quality may be different across these settings. In a recent white paper on observation care published by the Society of Hospital Medicine (SHM) Public Policy Committee, ${ }^{7}$ the SHM reported the results of a 2017 survey of its members about observation care. The results were concerning. An overwhelming majority of respondents (87\%) believed that the rules for observation are unclear for patients, and $68 \%$ of respondents believed that policy changes mandating informing patients of their observation status have created conflict between the provider and the patient.7 As shared by one respondent, "the observation issue can severely damage the therapeutic bond with patient/ family, who may conclude that the hospitalist has more interest in saving someone money at the expense of patient care."7 Thus, there is significant concern about the nature of observation stays and the experience for patients and providers. We should take care to better understand these experiences given 
that readmission reduction efforts may funnel more patients into observation care.

As a next step, we recommend further examination of how "revisit" rates have changed over time for patients with any discharge diagnosis, and not just those with pneumonia, AMI, or $H F^{8}$ Such examinations should be stratified by payer to identify differential impacts on those with lower socioeconomic status. Analyses should also examine changes in "revisit" types at the hospital level to better understand if hospitals with reductions in readmission rates are simply shifting revisits to the observation unit or ED. It is possible that inpatient readmissions for any given hospital are decreasing without concomitant increases in observation visits, as there are forces independent of the readmission penalty, such as the Recovery Audit Contractor program, that are driving hospitals to more frequently code patients as observation visits rather than inpatient admissions. ${ }^{9}$ Thus, readmissions could decrease and observation unit visits

\section{References}

1. Smith M, Saunders R, Stuckhardt L, McGinnis JM, eds. Best care at lower cost: the path to continuously learning health care in America. Washington, DC: National Academies Press; 2013.

2. Nuckols TK, Fingar KR, Barrett ML, et al. Returns to emergency department observation, or inpatient care within 30 days after hospitalization in 4 states, 2009 and 2010 versus 2013 and 2014. J Hosp Med. 2018;13(5):296-303.

3. Fingar KR, Washington R. Trends in Hospital Readmissions for Four High-Volume Conditions, 2009-2013. Statistical Brief No. 196. https://www.hcup-us. ahrq.gov/reports/statbriefs/sb196-Readmissions-Trends-High-Volume-Conditions.pdf. Accessed March 5, 2018.

4. Zuckerman RB, Sheingold SH, Orav EJ, Ruhter J, Epstein AM. Readmissions, observation, and the Hospital Readmissions Reduction Program. N Engl J Med. 2016;374(16):1543-1551. DOI: 10.1056/NEJMsa1513024

5. Gerhardt G, Yemane A, Apostle K, Oelschlaeger A, Rollins E, Brennan N Evaluating whether changes in utilization of hospital outpatient services contributed to lower Medicare readmission rate. Medicare Medicaid Res Rev. 2014;4(1). DOI: 10.5600/mmrr2014-004-01-b03 could increase independent of one another. We also recommend further research to examine differences in care quality, clinical outcomes, and costs for those readmitted to the hospital within 30 days of discharge versus those cared for in observation units or the ED. The challenge of such studies will be to identify and examine comparable populations of patients across these three settings. Examining patient perceptions and preferences across these settings is also critical. Finally, when assessing interventions to reduce inpatient readmissions, we need to consider "revisits" as a whole, not simply readmissions. ${ }^{10}$ Otherwise, we may simply be promoting the use of interventions that shift inpatient readmissions to observation unit or ED revisits, and there is little that is patient-centered or high value about that. ${ }^{9}$

Disclosures: The authors have nothing to disclose.

6. Kangovi S, Cafardi SG, Smith RA, Kulkarni R, Grande D. Patient financial responsibility for observation care. J Hosp Med. 2015;10(11):718-723. DOI: 10.1002/jhm.2436

7. The Hospital Observation Care Problem: Perspectives and Solutions from the Society of Hospital Medicine. Society of Hospital Medicine Public Policy Committee. https://www.hospitalmedicine.org/globalassets/policy-and-advocacy/advocacy-pdf/shms-observation-white-paper-2017. Accessed February 12, 2018.

8. Rosen AK, Chen Q, Shwartz M, et al. Does use of a hospital-wide readmission measure versus condition-specific readmission measures make a difference for hospital profiling and payment penalties? Medical Care. 2016;54(2):155161. DOI: 10.1097/MLR.0000000000000455.

9. Baugh CW, Schuur JD. Observation care-high-value care or a cost-shifting loophole? N Engl J Med. 2013;369(4):302-305. DOI: 10.1056/NEJMp1304493.

10. Cassel CK, Conway PH, Delbanco SF, Jha AK, Saunders RS, Lee TH. Getting more performance from performance measurement. $N$ Engl J Med. 2014;371(23):2145-2147. DOI: 10.1056/NEJMp1408345. 